Research Article

\title{
Uplink Resource Allocation for Interference Mitigation in Two-Tier Femtocell Networks
}

\author{
Sung-Yeop Pyun, ${ }^{1}$ Woongsup Lee $\mathbb{D}^{2},{ }^{2}$ and Ohyun Jo $\mathbb{D}^{3}$ \\ ${ }^{1}$ Korea Telecom, Seoul, Republic of Korea \\ ${ }^{2}$ Department of Information and Communication Engineering, Gyeongsang National University, Tongyeong 53064, \\ Republic of Korea \\ ${ }^{3}$ Department of Computer Science, College of Electrical and Computer Engineering, Chungbuk National University, \\ Cheongju 28644, Republic of Korea
}

Correspondence should be addressed to Ohyun Jo; jjo9804@gmail.com

Received 6 August 2018; Revised 8 October 2018; Accepted 31 October 2018; Published 2 December 2018

Guest Editor: Safdar H. Bouk

Copyright (C) 2018 Sung-Yeop Pyun et al. This is an open access article distributed under the Creative Commons Attribution License, which permits unrestricted use, distribution, and reproduction in any medium, provided the original work is properly cited.

\begin{abstract}
Two-tier femtocell networks, in which a large number of femto base stations (BSs) are deployed within a region overlapping with a macrocell, can provide an economical means of increasing user capacity and coverage. Given that femto BSs may be deployed with no cell planning, cross-tier interference generated from a number of macrocells and femtocells can cause severe problems. In particular, a macro mobile station (MS) that transmits uplink data may generate severe interference with adjacent femtocells, which causes performance degradation. In order to solve these problems, two novel resource allocation schemes, optimization and heuristic, are proposed, which efficiently reduce uplink interference in two-tier femtocell networks. Simulation results at the system level verify that both proposed schemes can improve the average capacity of the femtocells, but the heuristic scheme outperforms the optimization scheme in terms of computational complexity.
\end{abstract}

\section{Introduction}

With the increasing popularity of high data rate wireless services, a number of communication techniques have been proposed to cope with the increase in mobile traffic. One simple yet powerful means of increasing the capacity of wireless networks involves decreasing the size of the cell because a small cell is perfectly adequate for providing high data rate services for multiple users through more efficient wireless environments. Accordingly, femtocells, which have a small cell coverage, have been regarded as a key element in providing high-quality services in homes or small offices [1].

Femto base stations (BSs) are low-transmit power BSs with restricted indoor service coverage. Femto BSs provide connections between mobile devices and service providers using a licensed band [2]. They provide an efficient way of achieving a high data rate and increased service area, especially in places where radio connection would otherwise not be supportable. In two-tier femtocell networks, a large number of femtocells may be overlapping in the service area of a conventional macrocell, which has a large service coverage.

Given that femtocells are usually deployed without any cell planning, macrocells and femtocells can interfere with each other, probably leading to severe performance degradation in two-tier femtocell networks, especially if the spectrum is shared among femtocells and macrocells $[3,4]$. For example, a mobile station (MS) of a macro BS that transmits with a high power or is located near the femto BS may cause severe uplink interference with neighboring femtocells, and accordingly, the capacity of the femtocells can deteriorate.

The importance of uplink interference in two-tier femtocell networks means that its mitigation has been extensively investigated in the previous literature [5-7]. Uplink capacity in CDMA-based two-tier femtocell networks was analyzed in [5], in which a technique for avoiding interference using sectorization and CDMA hopping was also presented. In [6], the uplink capacity of TDMA-based 
two-tier femtocell networks was analyzed by distinguishing between those cases where macro- and femtocells share the same frequency and those where the frequency is divided. When the frequency is divided, the interference generated between macrocells and femtocells may not occur when the spectral efficiency is reduced. However, when the frequency is shared, the uplink throughput may be increased by the proper allocation of transmit power. In [7], an uplink power control scheme was proposed, in which the maximum transmit power of femtocell users is adjusted, in an effort to mitigate cross-tier interference at a macro BS. Finally, efficient power control schemes in dense and smallcell networks are studied in $[4,8,9]$. The interference management algorithms for improvement of practical LTE and LTE-A mobile networks are studied and adopted practically as shown in [10-15].

We present efficient resource allocation algorithms that can reduce uplink interference in two-tier femtocell networks. To this end, we formulate an optimization scheme using integer programming (IP), but this turns out to be somewhat impractical due to its complexity. In order to provide a practical solution, we propose a heuristic scheme with low complexity in which femtocells and a macrocell perform resource allocation cooperatively.

\section{System Model}

We consider two-tier femtocell networks in which a set of femto $\mathrm{BSs} \mathbf{K}=\{1, \ldots, K\}$ are deployed in the coverage of a macro MB. Both macro BS and femto BSs use the same frequency band, and the bandwidths of the macro BS and the femto $\mathrm{BS}_{k}$ are denoted by $W$ and $W_{k}$, respectively. A set of macro MSs $\mathbf{M}=\{1, \ldots, M\}$ communicate with the macro BS. A set of femto MSs $\mathbf{M}_{k}=\left\{1, \ldots, M_{k}\right\}$ communicate with the femto $\mathrm{BS}_{k}$, where $k=1, \ldots, K$ that denotes the index of the femto BS.

Each frame containing downlink and uplink subframes has the same duration, where $t=1,2, \ldots$ is the index of the frame. Each uplink subframe is divided into a set $\mathbf{N}=\{1, \ldots, N\}$ of time slots, each of which has a fixed interval. Each femto BS may be synchronized with the overlay macro BS in the frames using GPS or the IEEE 1588 PTP (Precision Time Protocol) [16]. A quasistatic flat fading channel is assumed, in which the channel state is constant within a unit frame duration and can vary frame by frame. A BS is made aware of the state of the channels from channel feedback information.

The signal to interference/noise ratio (SINR) of femto $\mathrm{MS}_{i}$ belonging to femto $\mathrm{BS}_{k}$ in slot $j$ at time $t$ is given by

$$
\gamma_{i j}^{k}(t)=\frac{p_{i}^{k}(t) h_{i}^{k}(t)}{W_{k} N_{0}+I_{j}^{k}(t)},
$$

where $p_{i}^{k}(t)$ is the transmitted power of femto $\mathrm{MS}_{i}$ belonging to femto $\mathrm{BS}_{k}, h_{i}^{k}(t)$ is the gain experienced in the channel between femto $\mathrm{MS}_{i}$ and femto $\mathrm{BS}_{k}$, and $N_{0}$ denotes the amount of noise per hertz.

The amount of interference in the uplink of femto $\mathrm{BS}_{k}$ in slot $j$ at time $t$ is described as

$$
\begin{aligned}
I_{j}^{k}(t) & =I_{\text {macro } \longrightarrow j}^{k}+I_{\text {femto } \longrightarrow j}^{k}, \\
I_{\text {macro } \longrightarrow j}^{k} & =\sum_{m \in M} p_{m}(t) h_{m k}(t) b_{m j}(t), \\
I_{\text {femto } \longrightarrow j}^{k} & =\sum_{k^{\prime} \in K, k^{\prime} \neq k} \sum_{i^{\prime} \in M_{k^{\prime}}} p_{i^{\prime}}^{k^{\prime}}(t) h_{i^{\prime} k}^{k^{\prime}}(t) b_{i^{\prime} j}^{k^{\prime}}(t),
\end{aligned}
$$

where $I_{\text {macro }}^{k} \longrightarrow j$ is the amount of interference from the macro MSs to femto $\mathrm{BS}_{k}$ where $p_{m}(t)$ is the transmitted power of macro MS $m, h_{m k}(t)$ is the gain experienced in the channel between macro MS $m$ and femto $\mathrm{BS}_{k}$, and $b_{m j}(t)$ is the indicator of resource allocation for the macro BS. If the slot $j$ at frame $t$ is allocated for macro MS $m, b_{m j}(t)$ is 1 ; otherwise, it is 0 .

$I_{\text {femto } \longrightarrow j}^{k}$ is the amount of interference from the MSs located in the other femto BSs with femto $\mathrm{BS}_{k}$ where $p_{i^{\prime}}^{k^{\prime}}(t)$ is the transmitted power of femto MS $i^{\prime}$ in the femto BS $k^{\prime}$, $h_{i^{\prime} k}^{k^{\prime}}(t)$ is the gain experienced in the channel between femto MS $i^{\prime}$ located in femto $\mathrm{BS}_{k^{\prime}}$ and femto $\mathrm{BS}_{k}$, and $b_{i^{\prime} j}^{k^{\prime}}(t)$ is the indicator of resource allocation for femto $\mathrm{BS}_{k^{\prime}}$. If the slot $j$ at frame $t$ is allocated to femto MS $i^{\prime}, b_{i^{\prime} j}^{k^{\prime}}(t)$ is 1 ; otherwise, it is 0 . Given that the interference between femtocells can be mitigated using either transmit power control or fractional frequency reuse [17], the interference generated in the uplink between macro MSs and femtocells can be minimized.

Using uplink open-loop transmit power control [18], which was devised to compensate the propagation loss and channel fluctuation due to shadowing, a BS determines the transmit power of subordinated MSs such that the target SINR can be satisfied. Therefore, the transmitted power of the subordinated MSs can be estimated by the macro/femto BSs. The MS $i$ 's target SINR, $\gamma_{i}^{\text {target }}(t)$, can be determined from the MS's rate requirement.

\section{Efficient Resource Allocation}

Figure 1 shows how the BS operates under the proposed schemes. A scheduler conventionally selects a number of MSs from those connected to the BS and then decides on the number of slots for the selected MSs. Note that a variety of scheduling policies can be chosen, as in the conventional scheduler. The proposed schemes are then used to determine which time slots are allocated for the selected MSs.

$r_{m}(t)$ and $r_{i}^{k}(t)$ denote the number of time slots allocated to macro MS $m$ and femto MS $i$ located in femto $\mathrm{BS}_{k}$, respectively. Then, $R(t)=\sum_{m \in \mathbf{M}} r_{m}(t)$ and $R^{k}(t)=\sum_{i \in \mathbf{M}_{k}} r_{i}^{k}(t)$ are defined by the number of time slots allocated by a macro BS and femto $\mathrm{BS}_{k}$, respectively, where $0 \leq R(t) \leq N$ and $0 \leq R^{k}(t) \leq N$. The uplink resource utilization of macro BS $\lambda(t)$ and femto $\mathrm{BS}_{k} \lambda^{k}(t)$ can be expressed as

$$
\begin{aligned}
\lambda(t) & =\frac{R(t)}{N}, \quad \text { where } 0 \leq \lambda(t) \leq 1, \\
\lambda^{k}(t) & =\frac{R^{k}(t)}{N}, \quad \text { where } 0 \leq \lambda^{k}(t) \leq 1 \text { for } \quad k=1, \ldots, K,
\end{aligned}
$$

which is the number of allocated time slots as a proportion of the total time slots. 


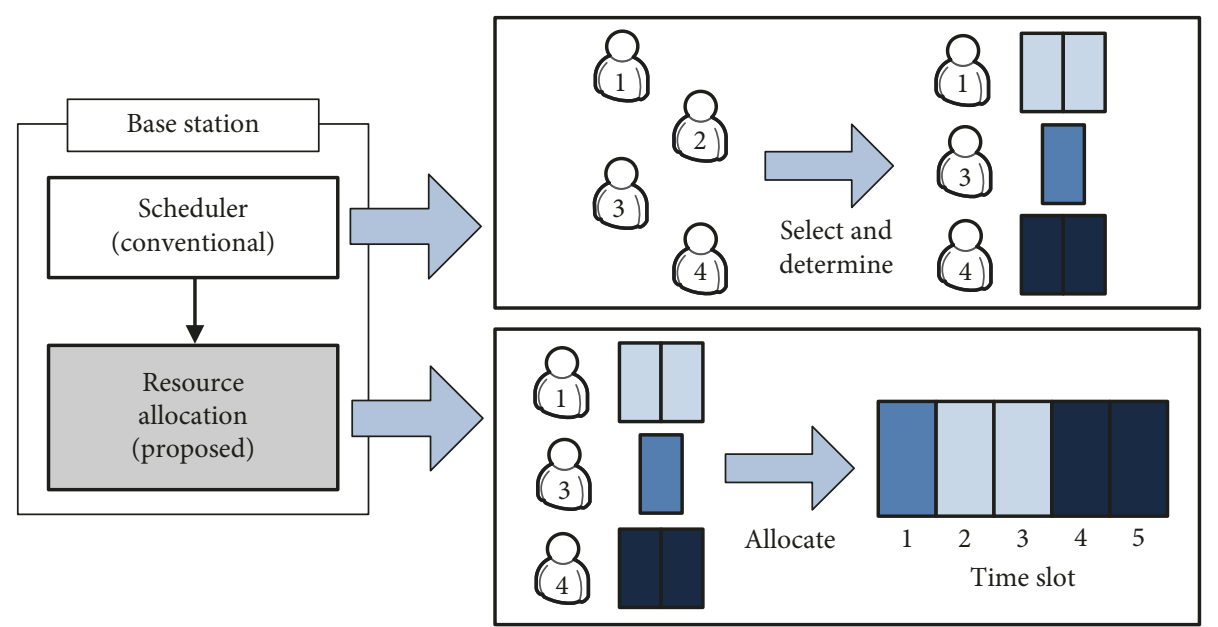

FIgURE 1: BS operation including scheduler and resource allocation. The term BS refers both macro and femto BSs.

3.1. Resource Allocation Using Optimization Scheme. In the optimization scheme, the uplink interference from macro MSs is supposed to be known to each femto $\mathrm{BS}_{k}$ through its backhaul link. Therefore, the optimization scheme can maximize the capacity of femto $\mathrm{BS}_{k}$ regardless of any resource allocation of a macro BS, $b_{m j}(t)$. We devise the optimization scheme using the following integer programming method:

$$
\max _{\mathbf{b}_{k}} \sum_{i \in \mathbf{M}_{k}} \sum_{j \in \mathbf{N}} b_{i j}^{k}(t) \log _{2}\left(1+\gamma_{i j}^{k}(t)\right)
$$

subject to

$$
\begin{aligned}
\sum_{i \in \mathbf{M}_{k}} b_{i j}^{k}(t) \leq 1, \quad \forall j \in \mathbf{N}, \\
\sum_{j \in \mathbf{N}} b_{i j}^{k}(t)=r_{i}^{k}(t), \quad \forall i \in \mathbf{M}_{k}, \\
b_{i j}^{k}(t) \in\{0,1\}, \quad \forall i \in \mathbf{M}_{k}, \quad j \in \mathbf{N}, \\
b_{m j}(t) \in\{0,1\}, \quad \forall m \in \mathbf{M}, \forall j \in \mathbf{N},
\end{aligned}
$$

where $\mathbf{b}_{k}$ is an $M_{k} \times N$ matrix, the elements of which denote the resource allocators of the femto $\mathrm{BS}_{k}, b_{i j}^{k}(t)$. The objective function (4) is intended to maximize the total sum capacity of all femto MSs belonging to the femto $\mathrm{BS}_{k}$. Moreover, equation (5) describes the constraint that a single time slot cannot be shared for multiple femto MSs simultaneously. Furthermore, equation (6) indicates that the total number of allocated time slots of femto MS $i$ is $r_{i}^{k}(t)$. Equations (7) and (8), respectively, describe the binary resource allocator of femto $\mathrm{BS}_{k} b_{i j}^{k}(t)$ and macro BS $b_{m j}(t)$.

From the solution of the formulated problem, each femto $\mathrm{BS}_{k}$ can obtain the optimal resource allocator $\mathbf{b}_{k}$. However, a huge signaling overhead is required to ascertain the uplink interference $I_{j}^{k}(t)$ at the femto BS. In addition, the femto BS needs to solve an integer programming problem, which also entails a large number of computations.
2.2. Resource Allocation Using Heuristic Scheme. To avoid the problems encountered with the optimization scheme, a heuristic scheme is proposed, which reduces the computational complexity by operating in a distributed way. Also, in the conventional OFDM-based two-tier/multitier resource allocation algorithms which are adopted in standards, macro BS and femto BSs use the separated resources or frequency bands to avoid and mitigate intercell interference. In this manner, the network-wise capacity cannot be optimized. Also, the cooperation of macro BS and femto BSs is hardly possible in the practical deployment scenarios because the vendors of macro BS and the vendors of femto BSs are not same generally. Thus, it is very important that the resource management algorithms between macro BS and femto BSs should work in a distributed manner. The system model in which the proposed scheme is working is exactly the same to the system model of the optimization scheme. Given that a macro MS that uses a great amount of power when transmitting may severely interfere with neighboring femtocells; by using the proposed heuristic algorithm, femto BSs can cooperate with the overlaid macro BS in resource allocation.

We describe how the macro BS operates in the heuristic scheme. First, the index of time slot $j=1$ and the resource allocator of macro BS $b_{m j}(t)=0$ are initialized. Second, the macro BS classifies the MSs scheduled at time $t, \mathbf{M}^{\prime}$. Third, the macro BS allocates time slots to the scheduled macro MSs based on the transmit power in the descending order. The macro MS $m^{\prime}$ with the largest transmit power is chosen from the scheduled macro MSs, $\mathbf{M}^{\prime}$. A slot $j$ is then allocated for the chosen macro MS $m^{\prime}\left(b_{m^{\prime} j}(t)=1\right)$. Finally, the index of slot $j$ and the number of slots allocated to macro MS $m^{\prime}$, $r_{m^{\prime}}(t)$, are updated. The macro BS may continue allocating slots to the chosen macro MS $m^{\prime}$ in the ascending order of the slot index (from 1 to $N$ ) until the number of slots allocated to macro MS $m^{\prime}$ is satisfied $\left(r_{m^{\prime}}(t)=0\right)$. This operation is summarized in Algorithm 1.

The operation of femto $\mathrm{BS}_{k}$ in the heuristic scheme is described as follows. First, the index of time slot $j=N$ and the resource allocator of femto BS $b_{i j}^{k}(t)=0$ are initialized. 
(1) Parameter initialization

(i) $j=1$ : slot index

(ii) $b_{m j}(t)=0, \forall m \in \mathbf{M}, \forall j \in \mathbf{N}$ : resource allocator

(2) Compute scheduled macro MS set

(i) $\mathbf{M}^{\prime}=\left\{m \in \mathbf{M} \mid r_{m}(t)>0\right\}$

(3) Choose a macro MS $m^{\prime}$

(i) $m^{\prime}=\arg \max _{m \in M^{\prime}} p_{m}(t)$

(4) Time slot allocation for the macro MS $m^{\prime}$

(i) $b_{m^{\prime} j}(t)=1$

(5) Parameter update

(i) $j=j+1$ and $r_{m^{\prime}}(t)=r_{m^{\prime}}(t)-1$

(6) If $r_{m^{\prime}}(t)=0$, go to step (7); otherwise, go back to step (4)

(7) Update the macro MS scheduling set

(i) $\mathbf{M}^{\prime}=\mathbf{M}^{\prime}-m^{\prime}$

(8) If $\mathbf{M}^{\prime}=\varnothing$, stop; otherwise, go back to step (3)

Algorithm 1: Macro BS operation in the heuristic scheme.

Second, the femto BS classifies the femto MS scheduling set at time $t, \mathbf{M}_{k}^{\prime}$. Third, given that the macro BS allocates slots to the scheduled macro MSs based on the transmit power in the descending order, the femto BS allocates slots to the scheduled femto MS in the descending order of the time slot (from $N$ to 1 ) in order to minimize interference from macro MSs, as follows. Even if the amount of interference from macro MSs is the same, a femto MS with a low target SINR may experience more capacity degradation than a femto MS with a high target SINR. Therefore, femto MS $i^{\prime}$ with the smallest target SINR is chosen from the scheduled femto MSs, $\mathbf{M}_{k}^{\prime}$. The femto BS then assigns a slot $j$ for the chosen femto MS $i^{\prime}\left(b_{i^{\prime} j}^{k}(t)=1\right)$. Finally, the index of slot $j$ and the number of slots to be allocated to macro MS $i^{\prime} r_{i^{\prime}}^{k}(t)$ are updated. The femto BS may keep allocating slots to the chosen femto MS $i^{\prime}$ in the descending order of the slot (from $N$ to 1 ) until the number of slots allocated to femto MS $i^{\prime}$ is satisfied $\left(r_{i^{\prime}}^{k}(t)=0\right)$. The operation of a femto BS is summarized in Algorithm 2.

Using the heuristic scheme means that the capacity of the femtocells may be lower than in cases where the optimization scheme is used because resources are allocated heuristically. However, because the femtocells do not need to receive any information about the interference generated in the uplink of macro MSs, the heuristic scheme can operate in a distributed manner. The computational complexity of the heuristic scheme, which can be derived as $O\left(N M_{k}\right)$, is also much lower than that of the optimization scheme. Even though the macro BS requires additional computations whose complexity is $O(N M)$ for cooperative resource allocation, the overall computational complexity is low such that the heuristic scheme is potentially affordable and takes place in real time. And the mobile station which generates intercell interference significantly can be efficiently taken
(1) Parameter initialization

(i) $j=N$ : index of time slot

(ii) $b_{i j}^{k}(t)=0, \forall i \in \mathbf{M}_{k}, \forall j \in \mathbf{N}$ : resource allocator

(2) Compute scheduled macro MS set

(i) $\mathbf{M}_{k}^{\prime}=\left\{i \in \mathbf{M}_{k} \mid r_{i}^{k}(t)>0\right\}$

(3) Choose a femto MS $i^{\prime}$

$$
i^{\prime}=\arg \min _{i \in \mathbf{M}_{k}^{\prime}} \gamma_{i}^{\text {target }}(t)
$$

(4) Time slot allocation for femto MS $i^{\prime}$

(i) $b_{i^{\prime} j}^{k}(t)=1$

(5) Parameter update

(ii) $j=j-1$ and $r_{i^{\prime}}^{k}(t)=r_{i^{\prime}}^{k}(t)-1$

(6) If $r_{i^{\prime}}^{k}(t)=0$, go to (7); otherwise, go back to step (4)

(7) Update the femto MS scheduling set

(i) $\mathbf{M}_{k}^{\prime}=\mathbf{M}_{k}^{\prime}-i^{\prime}$

(8) If $\mathbf{M}_{k}^{\prime}=\varnothing$, stop; otherwise, go back to step (3)

Algorithm 2: Femto BS $\mathrm{BS}_{k}$ operation in the heuristic scheme.

account into the resource allocations for macro BS and femto BSs in a distributed manner.

\section{Simulation Results and Conclusions}

For our simulation environment, we consider a two-tier femtocell network where $K$ femto BSs are uniformly located in the coverage of a macro BS. In our performance evaluation, we only consider the capacity of femto BSs located in the outer area of a macrocell. In the simulation, a proportional fair scheduler was used for the conventional scheduler of a BS. The channel gains $\left(h_{i}^{k}(t), h_{m k}(t)\right.$, and $\left.h_{i k}(t)\right)$, and the location of the MSs, were decided in the simulations with reference to the scenario in [19]. The detailed parameters are summarized in Table 1. We found out the solution of the optimization problem which is described in equations (4)-(8) by using MATLAB and then compared to the performance of the proposed algorithm evaluated also by MATLAB simulations.

Figure 2 describes the average capacity of an outer femto BS according to the uplink resource utilization $\lambda^{k}(t)$ in the case where the uplink resource utilization of macro BS is $\lambda(t)=0.4$. The proposed schemes evidently yield capacity enhancement over a random scheme in which time slots are randomly allocated to femto MSs. When the uplink resource utilization of femto BS $\lambda^{k}(t)$ increases, it is obvious that the amount of interference in the uplink increases as well. As a consequence, the amount of capacity enhancement by the proposed schemes falls from $41 \%$ to $4 \%$ because the scheduling gain decreases. When the resource utilization of the femto BS is at a maximum $\left(\lambda^{k}(t)=1\right)$, the gain of the proposed schemes is at a minimum.

Figure 3 describes the average capacity of the outer femto BS according to the uplink resource utilization $\lambda^{k}(t)$ in the case where the uplink resource utilization of the macro BS is $\lambda(t)=0.6$. When the uplink resource utilization of macro BS 
TABLE 1: Simulation parameters.

Macro cell coverage

Femtocell coverage

Number of femto BSs $(K)$

Number of macro MSs $(M)$

Number of femto MSs in femto $\mathrm{BS}_{k}\left(M_{k}\right)$

System bandwidth of macro BS $(W)$

System bandwidth of femto BS $\left(W_{k}\right)$

Distance between macro BS and outer femto BS $600 \mathrm{~m}$

Number of time slots $(N)$

Target SINR of macro MSs

Target SINR of femto MSs

Max TX power of MSs

External wall loss of femtocells

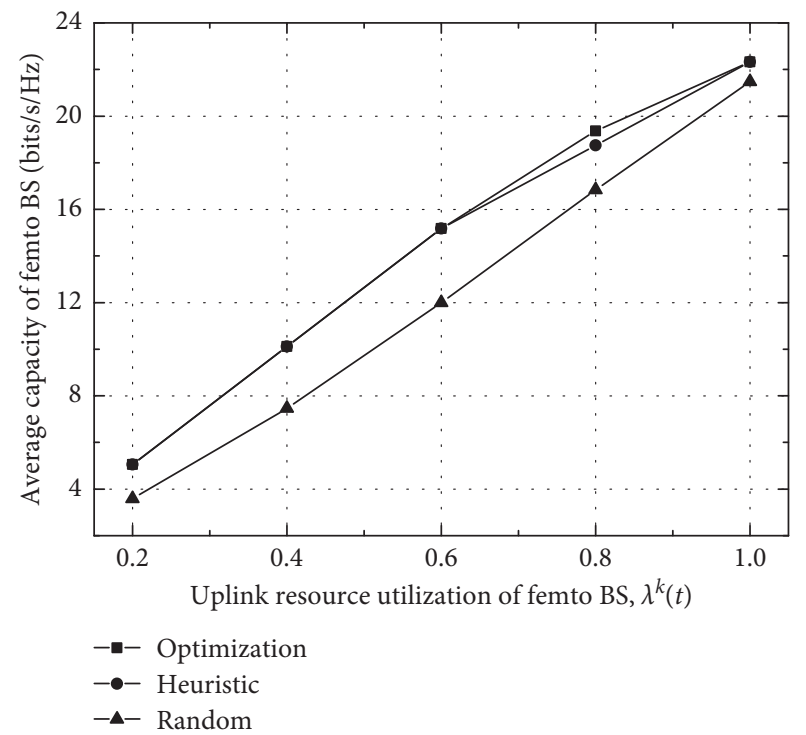

FIgURE 2: Average capacity of outer femto BS in the case where uplink resource utilization of macro BS $\lambda(t)=0.4$.

$\lambda(t)$ increases, the amount of uplink interference with the femto BSs also increases. As a consequence, the capacity enhancements of both heuristic scheme and random scheme decrease compared to those of the optimization scheme. When the resource utilization of femto $\mathrm{BS}_{k}$ is less than the unused resource utilization of the macro BS $\left(\lambda^{k}(t) \leq 1-\lambda(t)\right)$, the heuristic scheme can allocate the uplink resources that are not used by the macro MSs to femto MSs belonging to femto $\mathrm{BS}_{k}$. In this case, the heuristic scheme shows the same performance as the optimization scheme because it can avoid strong uplink interference.

Figure 4 describes the average capacity of the outer femto BS according to the uplink resource utilization $\lambda^{k}(t)$ in the case that the uplink resource utilization of macro BS is $\lambda(t)=0.8$. Even for the case that the resource utilization of a macro BS is higher, the proposed heuristic scheme still outperforms the random scheme.

Performance evaluation using intensive system level simulations verified that both proposed heuristic scheme and optimization scheme are efficient in improving the capacity of femtocells. The optimization scheme showed

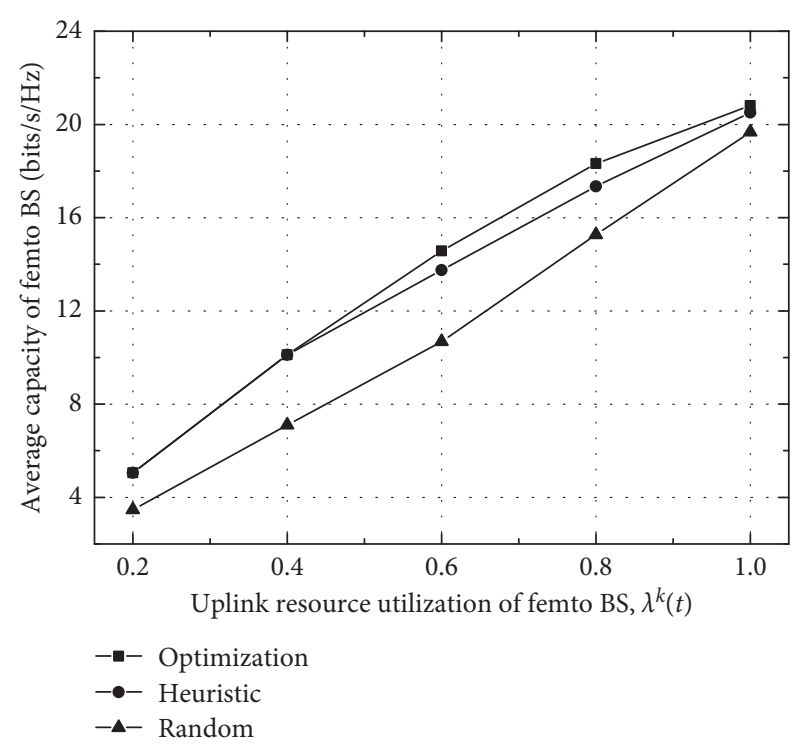

FIgURE 3: Average capacity of outer femto BS in the case where uplink resource utilization of macro BS $\lambda(t)=0.6$.

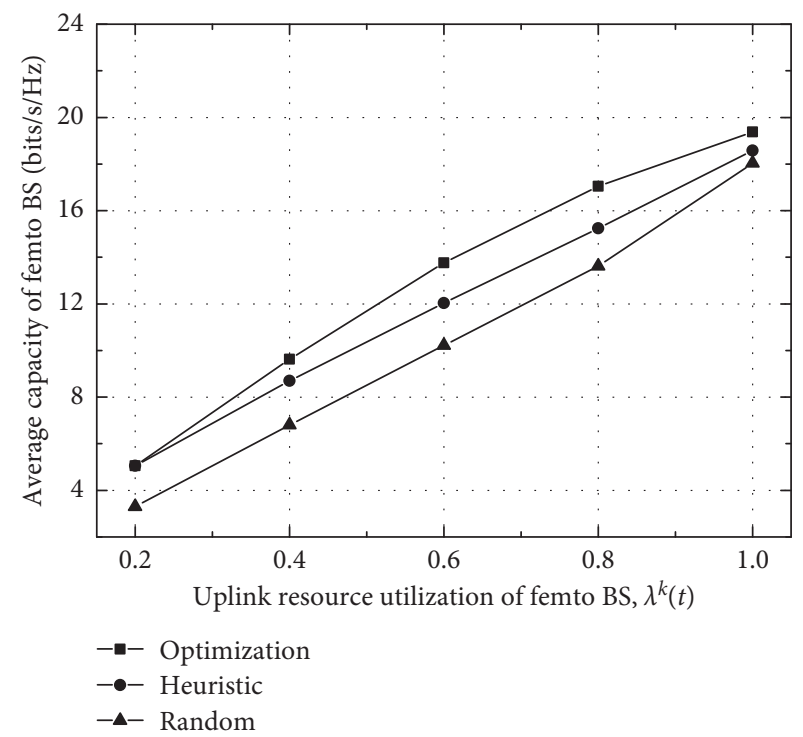

FIgURE 4: Average capacity of outer femto BS in the case where uplink resource utilization of macro BS $\lambda(t)=0.8$.

the best performance for all cases, and the performance of the heuristic scheme is comparable to that of the optimization scheme when the uplink resource utilization of the macro BS is lower. The heuristic scheme is more feasible in terms of its complexity for practical implementations because the femtocells do not need the additional procedure regarding the measurement and report of uplink interference from the macro MSs, which enables real-time implementation.

\section{Data Availability}

No data were used to support this study. 


\section{Conflicts of Interest}

The authors declare that they have no conflicts of interest.

\section{Acknowledgments}

This research was a part of the project titled Development of Distributed Underwater Monitoring and Control Networks, funded by the Ministry of Oceans and Fisheries, Korea. In part, this work was supported by the National Research Foundation of Korea (NRF) grant funded by the Korea Government (MSIT) (No. NRF-2018R1C1B5045013), and this work was supported by the research grant of Chungbuk National University in 2018.

\section{References}

[1] V. Chandrasekhar, J. Andrews, and A. Gatherer, "Femtocell networks: a survey," IEEE Communications Magazine, vol. 46, no. 9 , pp. 59-67, 2008.

[2] S. Pyun and D. Cho, "Resource allocation scheme for minimizing uplink interference in hierarchical cellular networks," in Proceedings of 2010 IEEE 71st Vehicular Technology Conference, Taipei, Taiwan, May 2010.

[3] J. Kim and D. Cho, "A joint power and subchannel allocation scheme maximizing system capacity in indoor dense mobile communication systems," IEEE Transactions on Vehicular Technology, vol. 59, no. 9, pp. 4340-4353, 2010.

[4] H. Zhang, S. Huang, C. Jiang, K. Long, V. C. M. Leung, and H. V. Poor, "Energy efficient user association and power allocation in millimeter wave based ultra dense networks with energy harvesting base stations," IEEE Journal on Selected Areas in Communications, vol. 35, no. 9, pp. 1936-1947, 2017.

[5] V. Chandrasekhar and J. Andrews, "Uplink capacity and interference avoidance for two-tier femtocell networks," IEEE Transactions on Wireless Communications, vol. 8, no. 7, pp. 3498-3509, 2009.

[6] R. S. Karlsson, "Radio resource sharing and capacity of some multiple access methods in hierarchical cell structures," in Proceedings of IEEE VTS 50th Vehicular Technology Conference, Amsterdam, Netherlands, September 1999.

[7] H. Jo, C. Mun, J. Moon, and J. Yook, "Interference mitigation using uplink power control for two-tier femtocell networks," IEEE Transactions on Wireless Communications, vol. 8, no. 10, pp. 4906-4910, 2009.

[8] H. Zhang, H. Liu, J. Cheng, and V. C. M. Leung, "Downlink energy efficiency of power allocation and wireless backhaul bandwidth allocation in heterogeneous small cell networks," IEEE Transactions on Communications, vol. 66, no. 4, pp. 1705-1716, 2018.

[9] H. Zhang, Y. Nie, J. Cheng, V. C. M. Leung, and A. Nallanathan, "Sensing time optimization and power control for energy efficient cognitive small cell with imperfect hybrid spectrum sensing," IEEE Transactions on Wireless Communications, vol. 16, no. 2, pp. 730-743, 2017.

[10] S. Mumtaz, J. Rodriguez, and I. Otung, "Smart resource allocation scheme for fair coexistence in LTE-U and WiFi," in Proceedings of 35th AIAA International Communications Satellite Systems Conference, , Trieste, Italy, October 2017.

[11] N. Abedini, B. Sadiq, and J. Li, "Uplink scheduling with power control command in an FDD half-duplex network," U.S. Patent, vol. 9, p. 949, 2018.
[12] R. Estrada Pico, Optimization models for resource management in two-tier cellular networks, Ph.D. thesis, Ecole de technologie superieure, 2014.

[13] D. Gonzalez, M. Garcia-Lozano, and S. R. Boque, "Intercell interference coordination for control channels in LTE and LTE-A: an optimization scheme based on evolutionary algorithms," Wireless Personal Communications, vol. 93, no. 3, pp. 687-708, 2017.

[14] C. Kosta, B. Hunt, A. U. Quddus, and R. Tafazolli, "On interference avoidance through inter-cell interference coordination (ICIC) based on OFDMA mobile systems," IEEE Communications Surveys and Tutorials, vol. 15, no. 3, pp. 973-995, 2013.

[15] D. Lee, G. Y. Li, and S. Tang, "Intercell interference coordination for LTE systems," IEEE Transactions on Vehicular Technology, vol. 62, no. 9, pp. 4408-4420, 2013.

[16] IEEE P802.16m/D12, DRAFT Amendment: Air Interface for Broadband Wireless Access Systems-Advanced Air Interface, IEEE, Piscataway, NJ, USA, 2011.

[17] H. Lee, D. Oh, and Y. Lee, "Mitigation of inter-femtocell interference with adaptive fractional frequency reuse," in Proceedings of IEEE International Conference on Communications, Cape Town, South Africa, May 2010.

[18] L. K. Tee, C. Van Rensburg, and J. Tsai, "Uplink power control for an OFDMA mobile cellular system," in Proceedings of IEEE 66th Vehicular Technology Conference, Baltimore, MD, USA, October 2007.

[19] T. Nihtila, "Increasing femto cell throughput with HSDPA using higher order modulation," in Proceedings of IEEE International Networking and Communications Conference, Lahore, Pakistan, May 2008. 


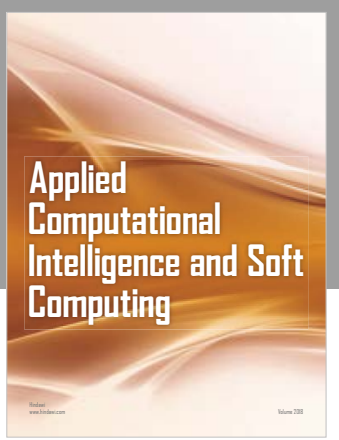

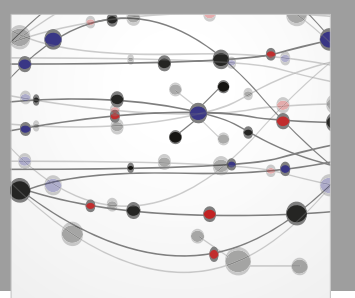

The Scientific World Journal
Submit your manuscripts at

Computing
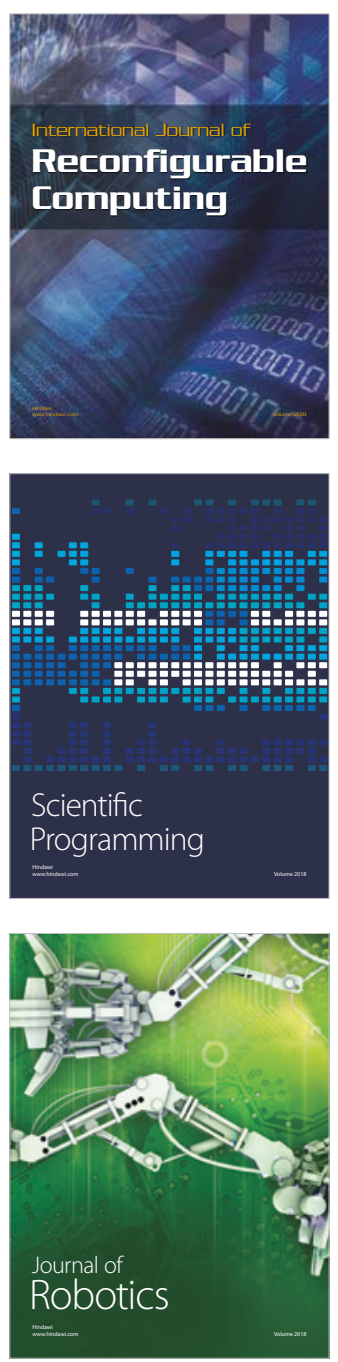

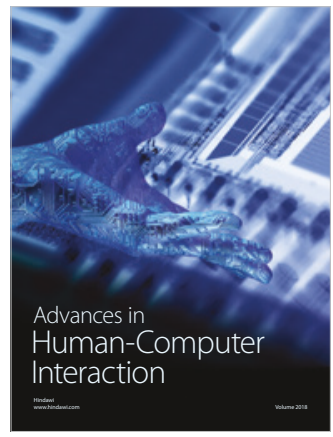

Human-Compute

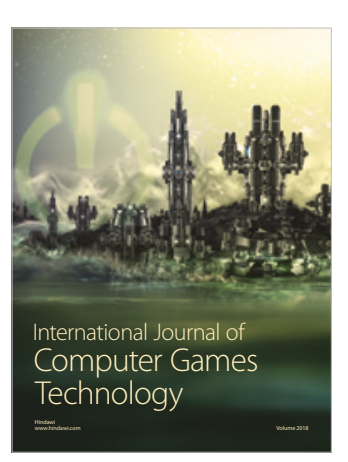

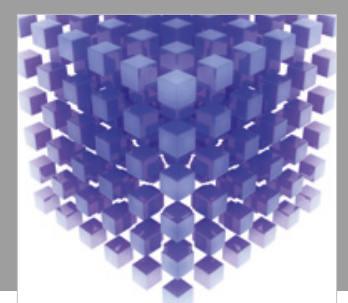

Mathematical Problems in Engineering

\section{Engincering}
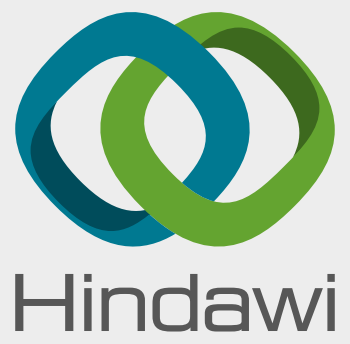

www.hindawi.com
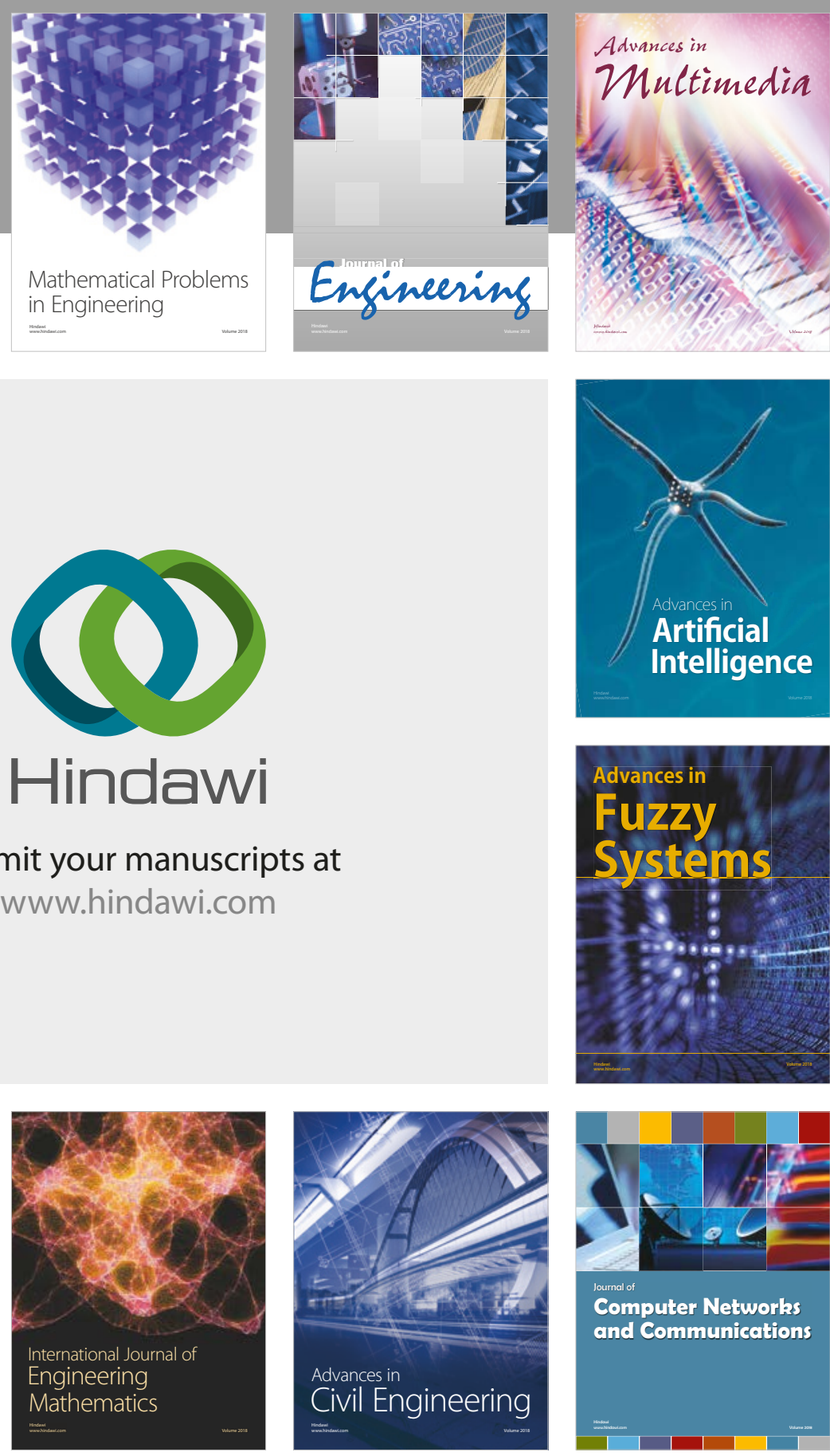

Computer Networks and Communications

Multimedia
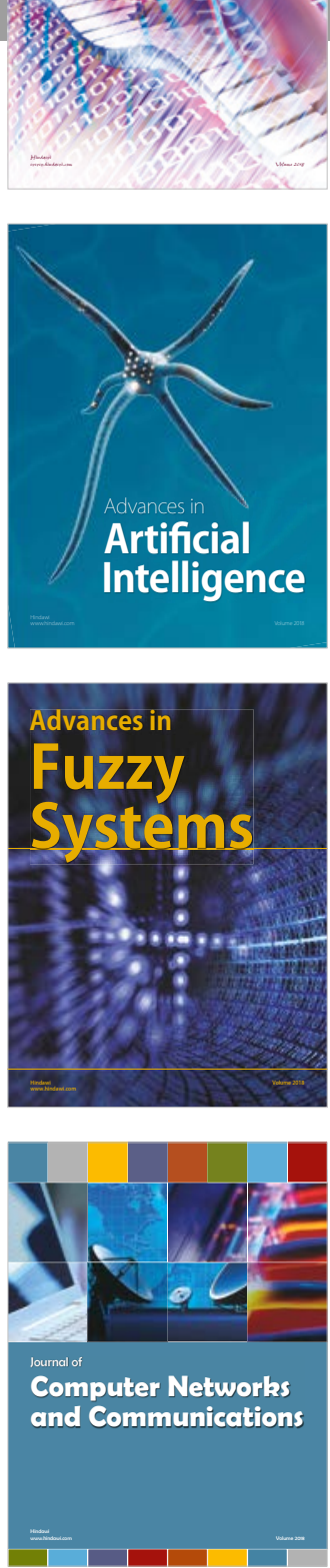

Advances in

Modelling \&

Simulation

in Engineering

interaction

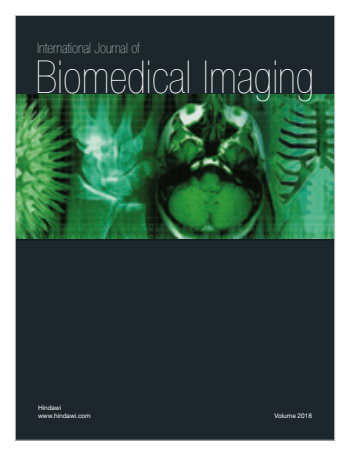

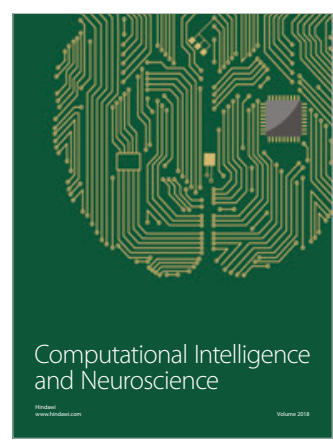

